\title{
A Wireless Multi Bundle Concentric Coil for Charging the Battery of a Total Artificial Heart or a Pacemaker
}

\author{
HM Amasha, ZK Ghazzawi, JI Al-Nabulsi
}

The Hashemite University, Zarka, Jordan

\begin{abstract}
One coil is placed outside the chest and is fed with an electromagnetic field, while monitoring the output on a specifically designed multi-bundle concentric coil to be implanted inside the body. It is assumed that the proposed coil should be easier to implant to the chest wall and is less prone to possible misalignments of the outer and internal coils, easier to isolate with a biocompatible material and most important, the feasibility of a much better heat dissipation scenario.

What type of waveform; triangular, square or sinusoidal, is best transformed from the outer coil to the inner on?. Furthermore, investigate the effect of different tissues separating the coils from each other.

Different coils with various radiuses and number of turns and shapes were tried in this experimental study. Results from the multi-bundle concentric coils were compared to results from single bundle coils and found to be better if not equal.
\end{abstract}

\section{Introduction}

Many considerations must be taken into account when implanting a device inside the human body; most important of which is to supply power to the device with minimal surgical intervention. Furthermore, in this case, the coil must not be bulky in size, easy to insulate with a bio-compatible material to enhance harmony with the surrounding tissues and reduce harm. It must, also, be flexible and easy to fix to the chest wall. The power transfer performance of the new proposed coil must not be less than the performance of existing coils [1]. A single-bundle coil (primary) is placed on the surface of the skin, while the multi-bundle coil (secondary) is placed under the skin opposing the primary coil. It is suggested to be as far as possible from the heart to eliminate interference, and as close to the skin surface as possible to decrease distance between the two coils. The primary is fed an electromagnetic signal with various frequencies and shapes to decide on the best frequency and shape in terms of power transfer and safety issues such as the values of the induced charging current and the levels of the dissipated heat. The internal coil could, also, report on the charging levels and the status of the implanted device.

\section{Methods}

The multi-bundle coil was constructed using a thin single wire wound 20 times in a bundle and then continues to be wound again in another similar bundle but with one centimeter larger radius and so on to form one coil with four concentric bundles, each having an increased two centimeters diameter from the previous one. Figure 1 shows a simulation of the field lines distribution when a lateral cut through the two coils. The primary single-bundle coil on the left and the secondary multi-bundle on the right spaced almost one centimeter apart.

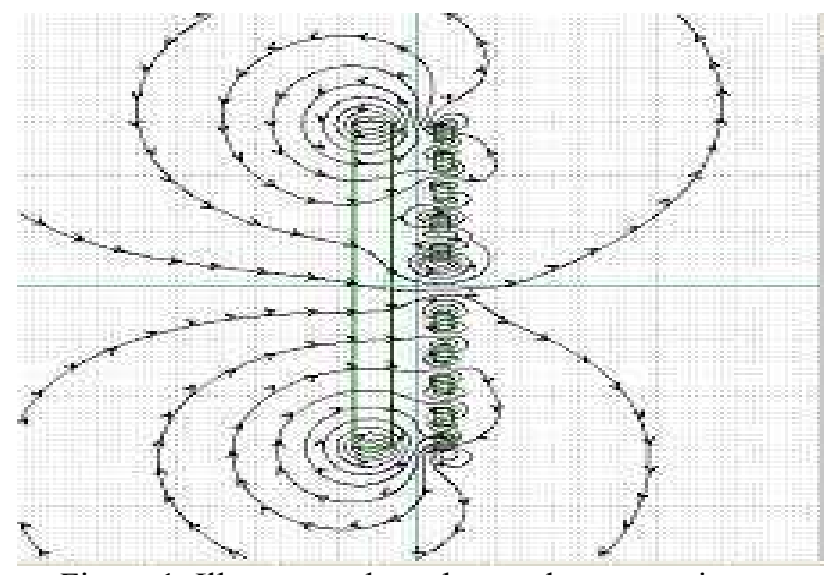

Figure 1: Illustrates a lateral central cross section through the two opposing coils and magnetic field lines.

An illustration of the multi-bundle coil is shown in Figure 2. Several coils of this shape has been constructed with varying number of turns in each bundle and tried under the same conditions.

Three shapes of signals were applied to the primary coil: rectangular, triangular and sinusoidal. All waveform 
were tested for whole of the safe frequency range applied in our experiments [2]. The frequencies ranged from 10 $\mathrm{kHz}$ up to $100 \mathrm{kHz}$ with steps of $10 \mathrm{kHz}$. The output of the secondary coil was connected to a rechargeable battery through a proper circuit and the charging curves were monitored $[3,4]$ as is shown in Figure 4.

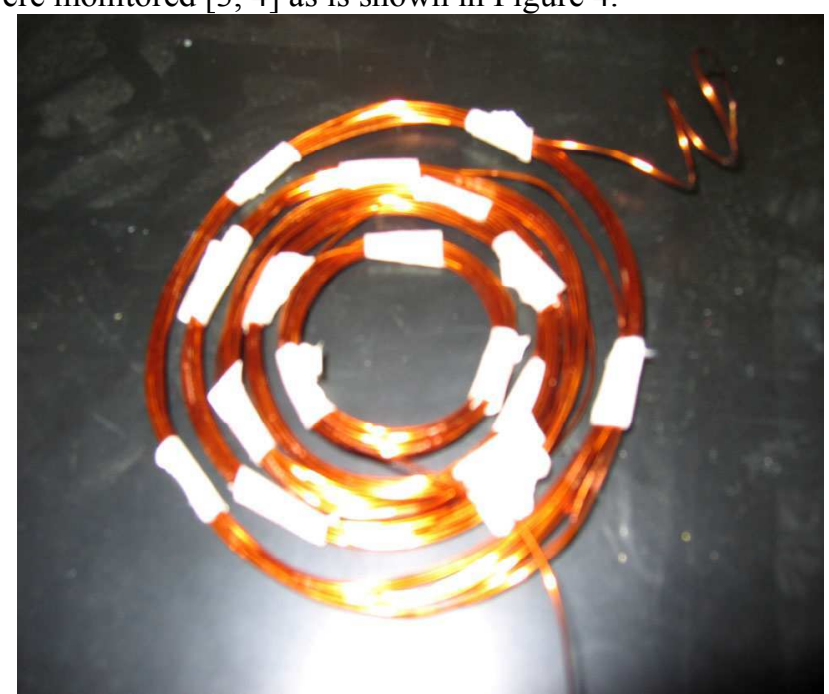

Figure 2: An experimental approximate figure showing a coil with only five bundles.

Three types of separators with varying thicknesses were inserted between the two coils; skin, fat and both. A two millimeter layer of skin, and $4 \mathrm{~mm}$ layer of fat alone, and then both later combined together. Normally, these layers are not as uniform in thickness and a layer of fat tends to accumulate between the bundles as time progresses. The location of implantation might introduce further anomalies in the thickness of these layers.

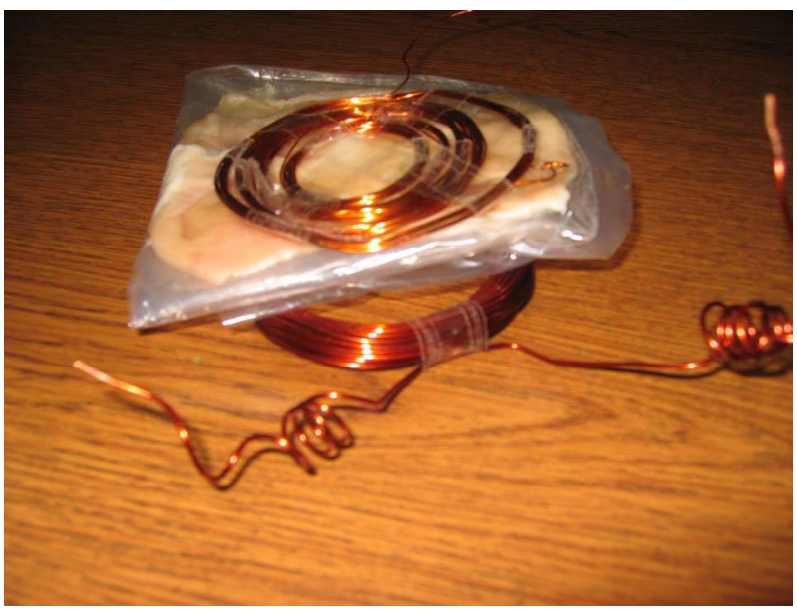

Figure 3: The primary (single-bundle) coil at the bottom separated by a $4 \mathrm{~mm}$-thick fat layer from the secondary (multi-bundle) coil on top.

\subsection{Finite Element Analysis on Coil Configuration}

In order to evaluate the thermal performance of the two coil configurations finite element analysis (FEA) is used. ANSYS ${ }^{\circledR}$ package is used for the analysis, where two dimensional models (2D) are made for the two coil configurations, one of which is shown in figure 4 , and another similar model is made for the single loop configuration.

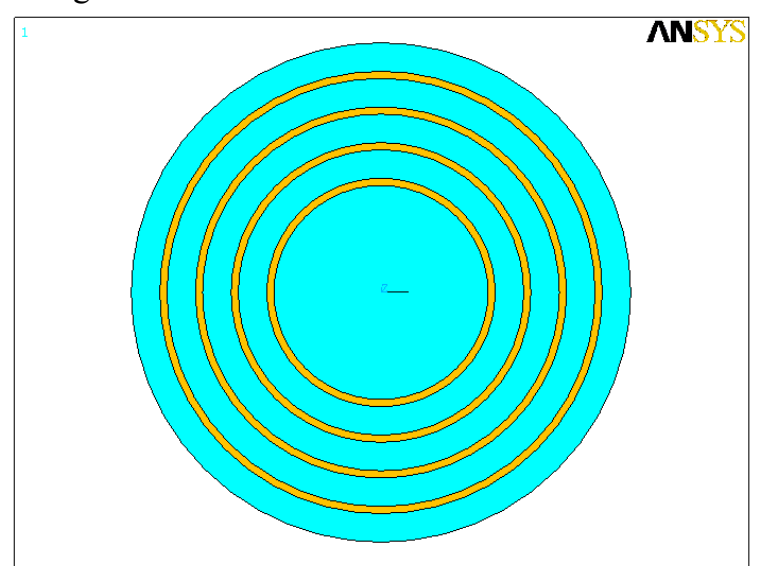

Figure 4: A 2D model for the multi-loop coil configuration.

Where the rings illustrated in figure 4 shows the copper loops and the remaining areas are skin adjacent to the coils. 2D triangular elements having 6 nodes are used for the meshing of the two models. Values of $400 \mathrm{~W} / \mathrm{m}^{2} . \mathrm{K}$ and $0.21 \mathrm{~W} / \mathrm{m}^{2} . \mathrm{K}$ are used respectively for copper and skin thermal conductivities.

The skin and copper regions of the model were then glued tighter in ANSYS ${ }^{\circledR}$ to ensure node connectivity at the interfaces.

A temperature of $310 \mathrm{~K}$ is assigned to the outer and inner skin areas of the model, and a heat flow of $5 \mathrm{~W}$ is assigned to the nodes within the copper loops.

\section{Results}

The results for the temperature distribution for the two models are shown in figures 5 and 6. 


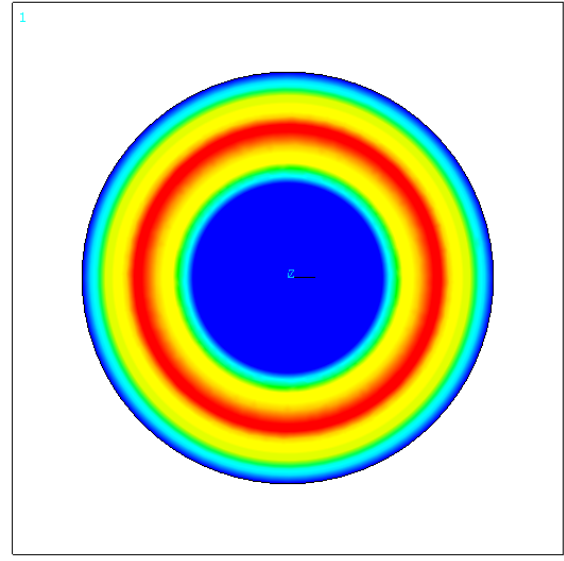

ANSYS 5.4 MAR 20.2007 $23: 43: 06$ NODAL SOI $\mathrm{SUB}=1$ TEMP (AVG) RSTS $=0$ PowerGraphics
EFACET $=1$ AVRES $=$ Mat SMN $=310$ $\mathrm{SMX}=310.327$ 만. 310 310.036
310.073 310.073
310.109 310.145
310.181 310.218
310.254 310.29

Figure 5: Temperature distribution for multi-loop coil configuration.

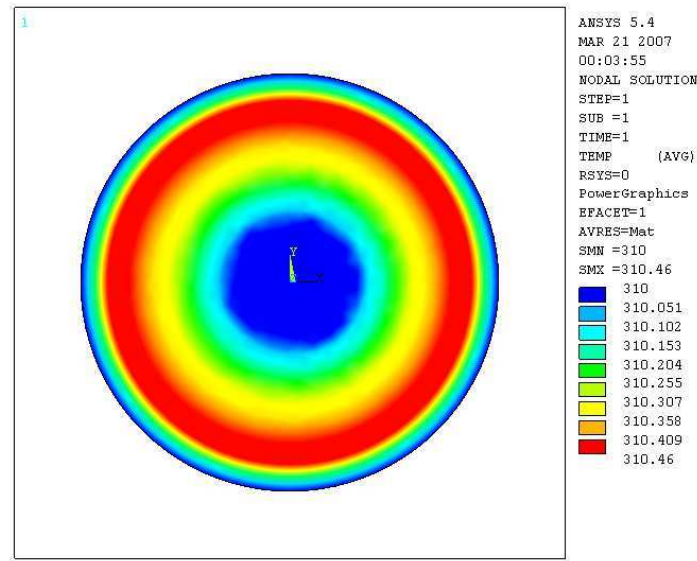

Figure 6: Temperature distribution for single-loop coil configuration.

The results showed a maximum increase in skin temperature of $0.35 \mathrm{C}$ for the multi-loop configuration and a $0.5 \mathrm{C}$ for the single-loop configuration. This highlight the advantage of using a multi-loop coil compared to a single loop, while keeping in mind that in biological systems a difference of $0.15 \mathrm{C}$ rise in temperature is significant.

Figures 7 and 8 show the thermal gradient for the two coil configurations. Where is can be concluded that the multi loop system yielded a smaller thermal gradient, which is advantageous in order to reduce the heat flow from the coil into adjacent skin. In addition the multiloop configuration showed a more uniform heat flow and reduced thermal spot regions.

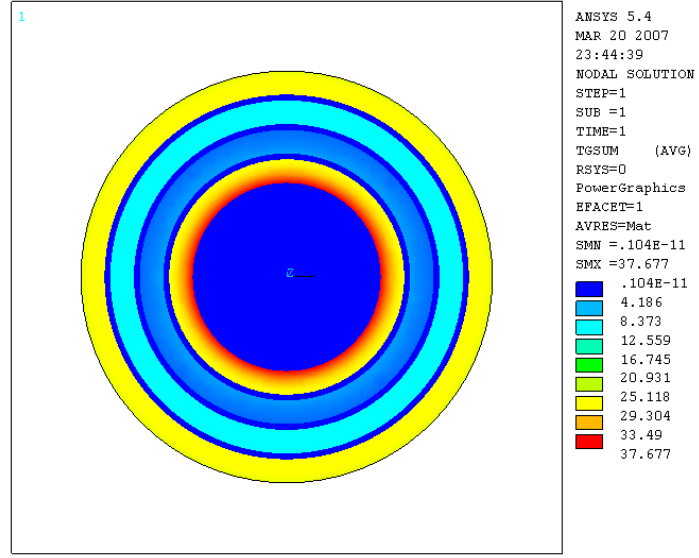

Figure 7: Thermal gradient for multi-loop coil configuration.

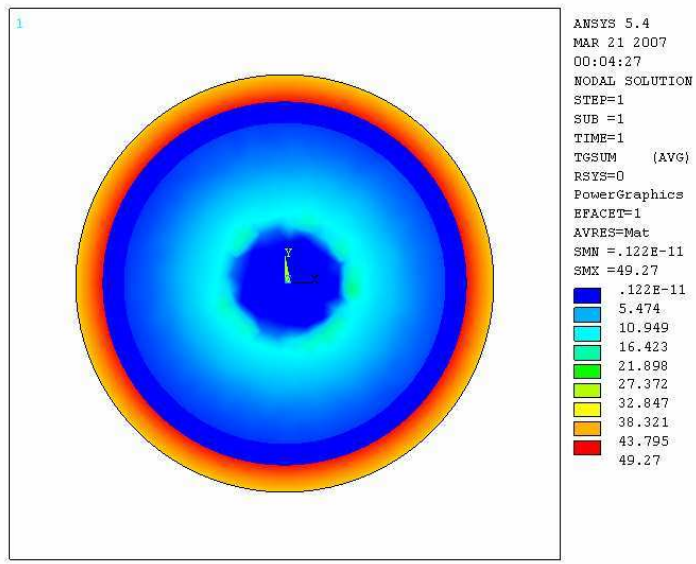

Figure 8: Thermal gradient for single-loop coil configuration.

The results obtained from the thermal analysis of the coils can be partly explained by the fact that the magnetic flux lines penetrating the multi-loop configuration are more distributed compared to the single-loop as shown in figure 1 , which would result in lower temperature rise in the multi-loop system, in addition to a lower thermal gradient.

Experiments showed that the results obtained on the multi-bundle coil were similar to those if we had a singlebundle coil as the secondary with the same total number of turns and a diameter equal to the diameter of the outermost bundle.

Results obtained using electromagnetic rectangular or triangular waveforms applied to the primary, did not always produce reliable values on the secondary and the output was not of a sufficient magnitude. Only the sinusoidal waveform produced enough current on the output to charge the battery without the need to increase the applied input to harmful levels $[2,3]$. 


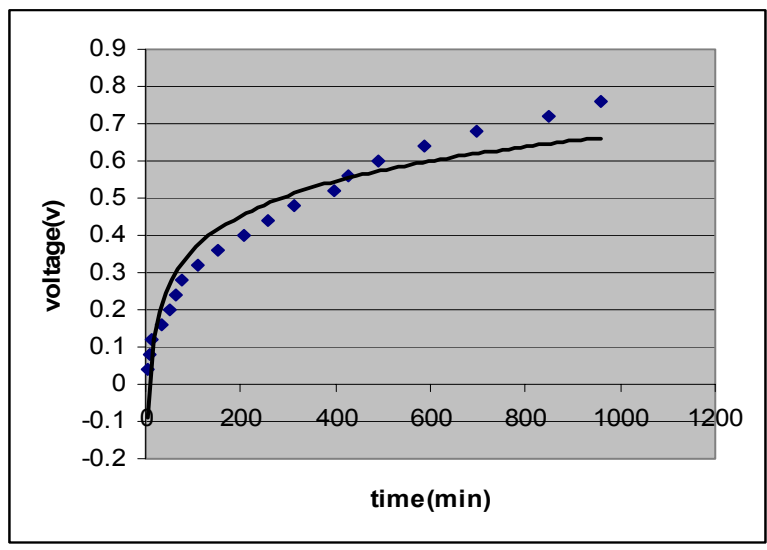

Figure 9: Charging curve of the battery.

Careful scanning of results collected at different frequencies indicates that we can choose a frequency around $20 \mathrm{kHz}$ without loosing much. In fact, in a few measurements, the results did not vary more than $5 \%$ across the whole selected range of study $(10-100 \mathrm{kHz})$. This would keep us well within the preferred zone of frequencies applied to the human body.

The output measured with skin alone compared to fat alone and when both combined together deteriorated, yet to an acceptable degree, partially because of the kind of material itself and also due to the increasing thicknesses. However, these results compare significantly to those when air or just a normal sheet of paper existed between the two coils.

The temperature distribution was measured using three thermistors at positions in the middle between each two bundles. One was placed outside the external bundle and one in the centre of the coils. Temperature was measured in case of a single bundle coils and in the case of the multi-bundle coil. The measured values for the multibundle coil were $65 \%$ of the measured temperature values near the single bundle. In vivo results may vary due to clearance by blood flow.

\section{Discussion and conclusions}

The proposed multi-bundle coil has shown comparable results to that of a single-bundle coil but it has advantages over it. Spacing between the bundles are preferable for two reasons; first, they allow for better isolation of each bundle with the biocompatible material, and second, these spaces allow for tissue to regenerate between these bundle and, hence, give better accommodation and fixation of the coil to the chest wall.

A very thin wire is being designed with higher number of turns per bundle is currently under the process of being implanted under the skin of a rabbit to test for the feasibility of using this coil.

\section{Acknowledgements}

Give any acknowledgements here.

\section{References}

[1] Hubert CL. Electrical machines, Theory, Operation, Application, Adjustments and Control. $2^{\text {nd }}$ Edition, Pearson Education, 2002.

[2] Webster JG. Medical Instrumentation and Design. $3^{\text {rd }}$ Edition, Wiley, 2001.

[3] Linden D, Reddy T. Handbook of Batteries. $3^{\text {rd }}$ Edition, McGraw-Hill Companies, 2002.

[4] Hayat WH, Kemmerly JE. Engineering Circuit Analysis. $5^{\text {th }}$ Edition, McGraw-Hill International Edition, 2003.

Address for correspondence

Dr Hani AMASHA

P.O.Box 961608,

Amman 11196,

Jordan

hamasha@hu.edu.jo

haniamasha@yahoo.com 